\title{
Phenomenological perspectives
}

\author{
Georg Weiglein
}

\author{
DESY \\ Lyon, 05 / 2013
}

D Determination of the properties of the new state at $\sim 126 \mathrm{GeV}$ : present and future

๑ What does the signal at $\sim 126 \mathrm{GeV}$ tell us?

D Where is the new physics that stabilises the gauge hierarchy?

- Conclusions 


\section{Determination of the properties of the new state}

\section{at $\sim 126 \mathrm{GeV}:$ present and future}

What do we know so far?

What can we find out in the future and how? 


\section{Determination of the properties of the state at $\sim 126 \mathrm{GeV}$}

Mass: statistical precision already remarkable with 2012 data

$\Rightarrow$ Need careful assessment of systematic effects for $\gamma \gamma$ and $Z Z^{*}$ channels, e.g. interference of signal and background, ...

Spin: Observation in $\gamma \gamma$ channel $\Rightarrow$ spin 0 or spin $2 ?$ 


\section{Determination of the properties of the state at $\sim 126 \mathrm{GeV}$}

Mass: statistical precision already remarkable with 2012 data

$\Rightarrow$ Need careful assessment of systematic effects for $\gamma \gamma$ and $Z Z^{*}$ channels, e.g. interference of signal and background, ...

Spin: Observation in $\gamma \gamma$ channel $\Rightarrow$ spin 0 or spin 2 ?

At which level of significance can the hypothesis spin $=1$ be excluded ( $2 \gamma$ 's vs. $4 \gamma$ 's)? 


\section{Determination of the properties of the state at $\sim 126 \mathrm{GeV}$}

Mass: statistical precision already remarkable with 2012 data

$\Rightarrow$ Need careful assessment of systematic effects for $\gamma \gamma$ and $Z Z^{*}$ channels, e.g. interference of signal and background, ...

Spin: Observation in $\gamma \gamma$ channel $\Rightarrow$ spin 0 or spin 2 ?

At which level of significance can the hypothesis spin $=1$ be excluded ( $2 \gamma$ 's vs. $4 \gamma$ 's)?

Spin can in principle be determined by discriminating between distinct hypotheses for spin 0, (1), 2 


\section{$\mathcal{C P}$ properties}

$\mathcal{C P}$-properties: experimentally much more difficult than spin Can be any admixture of $\mathcal{C P}$-even and $\mathcal{C P}$-odd components

Observables investigated up to now $\left(H \rightarrow Z Z^{*}, W W^{*}\right.$ and $H$ production in weak boson fusion) involve $H V V$ coupling

General structure of $H V V$ coupling (from Lorentz invariance):

$$
a_{1}\left(q_{1}, q_{2}\right) g^{\mu \nu}+a_{2}\left(q_{1}, q_{2}\right)\left[\left(q_{1} q_{2}\right) g^{\mu \nu}-q_{1}^{\mu} q_{2}^{\nu}\right]+a_{3}\left(q_{1}, q_{2}\right) \epsilon^{\mu \nu \rho \sigma} q_{1 \rho} q_{2 \sigma}
$$

Pure $\mathcal{C P}$-even state: $a_{1}=1, a_{2}=0, a_{3}=0$,

Pure $\mathcal{C P}$-odd state: $a_{1}=0, a_{2}=0, a_{3}=1$

However, in most BSM models $a_{3}$ would be loop-induced and heavily suppressed $\Rightarrow$ Realistic models often predict $a_{3} \ll a_{1}$

$\Rightarrow$ Observables involving $H V V$ coupling provide only limited sensitivity to effects of a $\mathcal{C P}$-odd component 


\section{$\mathcal{C P}$ properties}

Observables involving the $H V V$ coupling "project" to the $\mathcal{C P}$-even component of the observed state 


\section{$\mathcal{C P}$ properties}

Observables involving the $H V V$ coupling "project" to the $\mathcal{C P}$-even component of the observed state

The fact that we have observed the new state in the $Z Z^{*}$ and $W W^{*}$ channels (at a certain level of significance) already tells us that it is most likely not a pure $\mathcal{C} \mathcal{P}$-odd state 


\section{$\mathcal{C P}$ properties}

Observables involving the $H V V$ coupling "project" to the $\mathcal{C P}$-even component of the observed state

The fact that we have observed the new state in the $Z Z^{*}$ and $W W^{*}$ channels (at a certain level of significance) already tells us that it is most likely not a pure $\mathcal{C} \mathcal{P}$-odd state

$\Rightarrow$ Discrimination between the hypotheses of a pure $\mathcal{C P}$-even and a pure $\mathcal{C} \mathcal{P}$-odd state will be relatively easy 


\section{$\mathcal{C P}$ properties}

Observables involving the $H V V$ coupling "project" to the $\mathcal{C P}$-even component of the observed state

The fact that we have observed the new state in the $Z Z^{*}$ and $W W^{*}$ channels (at a certain level of significance) already tells us that it is most likely not a pure $\mathcal{C} \mathcal{P}$-odd state

$\Rightarrow$ Discrimination between the hypotheses of a pure $\mathcal{C P}$-even and a pure $\mathcal{C} \mathcal{P}$-odd state will be relatively easy

However, this will not be sufficient to determine the $\mathcal{C P}$ properties of the new state

Which upper limit on a $\mathcal{C P}$-odd admixture can be set? 


\section{$\mathcal{C P}$ properties}

Observables involving the $H V V$ coupling "project" to the $\mathcal{C P}$-even component of the observed state

The fact that we have observed the new state in the $Z Z^{*}$ and $W W^{*}$ channels (at a certain level of significance) already tells us that it is most likely not a pure $\mathcal{C} \mathcal{P}$-odd state

$\Rightarrow$ Discrimination between the hypotheses of a pure $\mathcal{C P}$-even and a pure $\mathcal{C} \mathcal{P}$-odd state will be relatively easy

However, this will not be sufficient to determine the $\mathcal{C P}$ properties of the new state Which upper limit on a $\mathcal{C P}$-odd admixture can be set?

$\Rightarrow$ Channels involving only Higgs couplings to fermions provide much higher sensitivity 


\section{Coupling determination}

\section{- What is meant by measuring a coupling?}




\section{Coupling determination}

- What is meant by measuring a coupling?

A coupling is not directly a physical observable; what is measured is $\sigma \times \mathrm{BR}$ (within acceptances), etc.

$\Rightarrow$ Need to specify a Lagrangian in order to define the meaning of coupling parameters 


\section{Coupling determination}

- What is meant by measuring a coupling?

A coupling is not directly a physical observable; what is measured is $\sigma \times \mathrm{BR}$ (within acceptances), etc.

$\Rightarrow$ Need to specify a Lagrangian in order to define the meaning of coupling parameters

- The experimental results that have been obtained for the various channels are not model-independent Properties of the SM Higgs have been used for discriminating between signal and background Need the SM to correct for acceptances and efficiencies 


\section{Coupling determination}

- What is meant by measuring a coupling?

A coupling is not directly a physical observable; what is measured is $\sigma \times \mathrm{BR}$ (within acceptances), etc.

$\Rightarrow$ Need to specify a Lagrangian in order to define the meaning of coupling parameters

- The experimental results that have been obtained for the various channels are not model-independent Properties of the SM Higgs have been used for discriminating between signal and background Need the SM to correct for acceptances and efficiencies

- The total Higgs width cannot be measured at the LHC without additional assumptions

$\Rightarrow$ Can in general only determine ratios of couplings, not absolute coupling values 


\section{Higgs coupling determination at the LHC}

Problem: no absolute measurement of total production cross section (no recoil method like LEP, ILC: $e^{+} e^{-} \rightarrow Z H$, $\left.Z \rightarrow e^{+} e^{-}, \mu^{+} \mu^{-}\right)$

Production $\times$ decay at the LHC yields combinations of Higgs couplings $\left(\Gamma_{\text {prod, decay }} \sim g_{\text {prod, decay }}^{2}\right)$ :

$$
\sigma(H) \times \operatorname{BR}(H \rightarrow a+b) \sim \frac{\Gamma_{\text {prod }} \Gamma_{\text {decay }}}{\Gamma_{\text {tot }}},
$$

Large uncertainty on dominant decay for light Higgs: $H \rightarrow b \bar{b}$

$\Rightarrow$ Without further assumtions, total Higgs width cannot be determined

$\Rightarrow$ LHC can directly determine only ratios of couplings, e.g. $g_{H \tau \tau}^{2} / g_{H W W}^{2}$ 


\section{Determination of couplings and $\mathcal{C} \mathcal{P}$ properties}

\section{need to be addressed together}

Deviations from the SM: in general both the absolute value of the couplings and the tensor structure of the couplings (affects $\mathcal{C P}$ properties) will change 


\section{Determination of couplings and $\mathcal{C P}$ properties}

\section{need to be addressed together}

Deviations from the SM: in general both the absolute value of the couplings and the tensor structure of the couplings (affects $\mathcal{C P}$ properties) will change

$\Rightarrow$ Determination of couplings and determination of $\mathcal{C P}$ properties can in general not be treated separately from each other 


\section{Determination of couplings and $\mathcal{C P}$ properties}

\section{need to be addressed together}

Deviations from the SM: in general both the absolute value of the couplings and the tensor structure of the couplings (affects $\mathcal{C P}$ properties) will change

$\Rightarrow$ Determination of couplings and determination of $\mathcal{C P}$ properties can in general not be treated separately from each other

Deviations from the SM would in general change kinematic distributions

$\Rightarrow$ No simple rescaling of $\mathrm{MC}$ predictions possible

$\Rightarrow$ Not feasible for analysis of 2012 data set

$\Rightarrow$ LHC Higgs XS WG: Proposal of "interim framework" 
Recommendations of the $L M$ subgroup of the LHC Higgs XS WG for analyses of 2012 data

Assumptions:

- Signal corresponds to only one state, no overlapping resonances, etc.

- Zero-width approximation

- Only modifications of coupling strenghts (absolute values of the couplings) are considered, no modification of the tensor structure as compared to the SM case

$\Rightarrow$ Assume that the observed state is a $\mathcal{C P}$-even scalar 
Recommendations of the $L M$ subgroup of the LHC Higgs XS WG for analyses of 2012 data

Use state-of-the-art predictions in the SM and rescale the predictions with "leading order inspired" scale factors $\kappa_{i}$ $\left(\kappa_{i}=1\right.$ corresponds to the SM case)

Note: scaling of couplings is in general not possible if higher-order electroweak corrections are included

In the SM: Higgs sector is determined by single parameter $M_{\mathrm{H}}$ (+ higher-order contributions)

$\Rightarrow$ Once $M_{\mathrm{H}}$ is fixed the Higgs couplings are determined and cannot be varied within the SM 
Recommendations of the $L M$ subgroup of the LHC Higgs XS WG for analyses of 2012 data

Which kind of scaling factors should be considered?

In general, scale factors are needed for couplings of the new state to

$t, b, \tau, W, Z, \ldots$

+ extra loop contribution to $\sigma(g g \rightarrow H), \Gamma(H \rightarrow g g)$

+ extra loop contribution to $\Gamma(H \rightarrow \gamma \gamma)$

+ additional contributions to total width, $\Gamma_{H}$, from undetectable final states

Total width $\Gamma_{H}$ cannot be measured without further assumptions (otherwise only coupling ratios can be determined, not absolute values of couplings) 


\section{Proposed "benchmarks" for scale factors $\kappa_{i}$}

Different "benchmark" proposals, based on simplifying assumptions to reduce the number of free parameters

1 parameter: overall coupling strength $\mu$

2 parameters: e.g. common scale factor $\kappa_{V}$ for $W, Z$, and common scale factor for all fermions, $\kappa_{F}$

For each benchmark (except overall coupling strength) two versions are proposed:

with and without taking into account the possibility of additional contributions to the total width 


\section{Proposed "benchmarks" for scale factors $\kappa_{i}$}

If additional contributions to $\Gamma_{H}$ are allowed

$\Rightarrow$ Determination of ratios of scaling factors, e.g. $\kappa_{i} \kappa_{j} / \kappa_{H}$

If no additional contributions to $\Gamma(H \rightarrow \gamma \gamma), \Gamma_{H}, \ldots$ are allowed

$\Rightarrow \kappa_{\gamma}$ can be determined in terms of $\kappa_{b}, \kappa_{t}, \kappa_{\tau}, \kappa_{W}$

evaluated to NLO QCD accuracy

Example: $\kappa_{V}, \kappa_{F}$ analyses from CMS and ATLAS
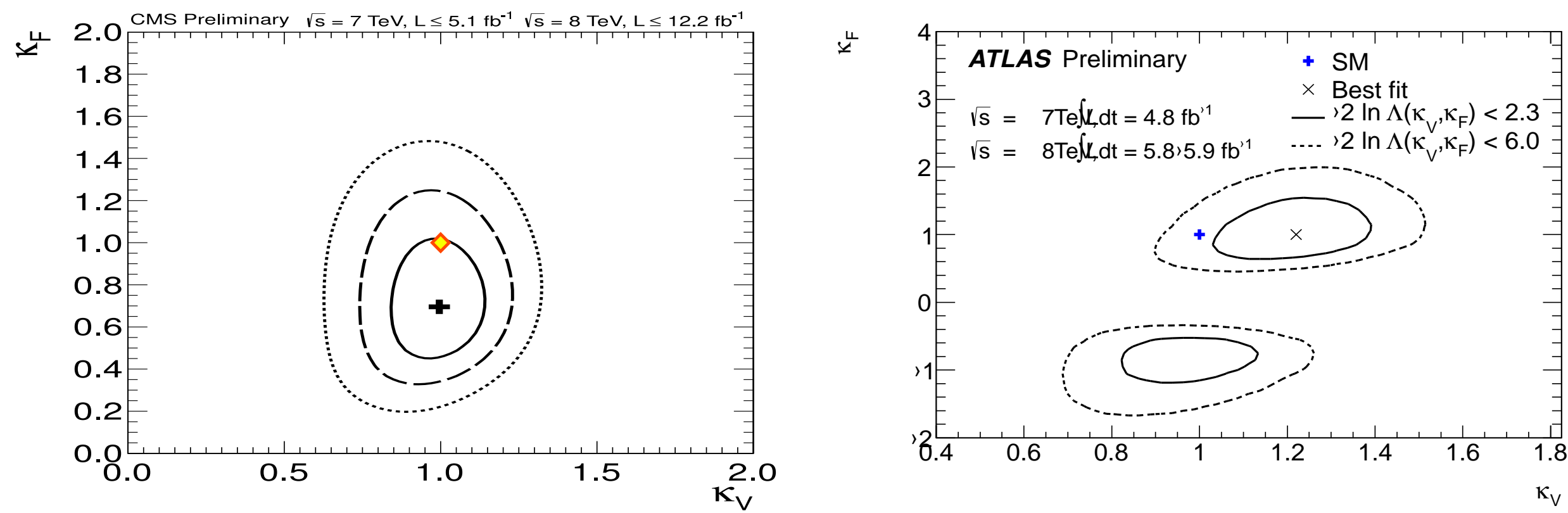


\section{How to interpret the results in terms of $\kappa_{i}$ ?}

- The $\kappa_{i}$ have been introduced to test the compatibility of the data with the SM The results cannot be interpreted as "coupling measurements"! 


\section{How to interpret the results in terms of $\kappa_{i}$ ?}

- The $\kappa_{i}$ have been introduced to test the compatibility of the data with the SM

The results cannot be interpreted as "coupling measurements"!

- The results for the $\kappa_{i}$ are obtained using various theoretical assumptions

Example: in a $\kappa_{V}, \kappa_{F}$ analysis only these two parameters are allowed to vary, while everything else is fixed to the SM value (+ assumption that acceptances and efficiencies are as in the SM + assumptions mentioned above) 


\section{How to interpret the results in terms of $\kappa_{i}$ ?}

- The $\kappa_{i}$ have been introduced to test the compatibility of the data with the SM

The results cannot be interpreted as "coupling measurements"!

- The results for the $\kappa_{i}$ are obtained using various theoretical assumptions

Example: in a $\kappa_{V}, \kappa_{F}$ analysis only these two parameters are allowed to vary, while everything else is fixed to the SM value ( + assumption that acceptances and efficiencies are as in the SM + assumptions mentioned above)

$\Rightarrow$ An analysis in terms of just $\kappa_{V}$ and $\kappa_{F}$ (or similar) would be much too restrictive in the future 


\section{How to interpret the results in terms of $\kappa_{i}$ ?}

- The $\kappa_{i}$ have been introduced to test the compatibility of the data with the SM

The results cannot be interpreted as "coupling measurements"!

- The results for the $\kappa_{i}$ are obtained using various theoretical assumptions

Example: in a $\kappa_{V}, \kappa_{F}$ analysis only these two parameters are allowed to vary, while everything else is fixed to the SM value ( + assumption that acceptances and efficiencies are as in the SM + assumptions mentioned above)

$\Rightarrow$ An analysis in terms of just $\kappa_{V}$ and $\kappa_{F}$ (or similar) would be much too restrictive in the future

$\Rightarrow$ It is not very useful to present projections for the HL-LHC in terms of $\kappa_{V}$ and $\kappa_{F}$, more general approach needed 


\section{Complementary approach: model-specific Higgs phenomenology beyond the SM}

Standard Model: a single parameter determines the whole Higgs phenomenology: $M_{\mathrm{H}}$

In the SM the same Higgs doublet is used "twice" to give masses both to up-type and down-type fermions

$\Rightarrow$ extensions of the Higgs sector having (at least) two doublets are quite "natural"

$\Rightarrow$ Would result in several Higgs states 


\section{Complementary approach: model-specific Higgs phenomenology beyond the SM}

Standard Model: a single parameter determines the whole Higgs phenomenology: $M_{\mathrm{H}}$

In the SM the same Higgs doublet is used "twice" to give masses both to up-type and down-type fermions

$\Rightarrow$ extensions of the Higgs sector having (at least) two doublets are quite "natural"

$\Rightarrow$ Would result in several Higgs states

Many extended Higgs theories have over large part of their parameter space a lightest Higgs scalar with properties very similar to those of the SM Higgs boson

Example: SUSY in the "decoupling limit" 


\section{Higgs physics in Supersymmetry}

"Simplest" extension of the minimal Higgs sector:

Minimal Supersymmetric Standard Model (MSSM)

- Two doublets to give masses to up-type and down-type fermions (extra symmetry forbids to use same doublet)

- SUSY imposes relations between the parameters 


\section{Higgs physics in Supersymmetry}

"Simplest" extension of the minimal Higgs sector:

Minimal Supersymmetric Standard Model (MSSM)

- Two doublets to give masses to up-type and down-type fermions (extra symmetry forbids to use same doublet)

- SUSY imposes relations between the parameters

$\Rightarrow$ Two parameters instead of one: $\tan \beta \equiv \frac{v_{u}}{v_{d}}, \quad M_{\mathrm{A}}$ (or $M_{\mathrm{H}^{ \pm}}$)

$\Rightarrow$ Upper bound on lightest Higgs mass, $M_{\mathrm{h}}$ : 


\section{Higgs physics in Supersymmetry}

"Simplest" extension of the minimal Higgs sector:

Minimal Supersymmetric Standard Model (MSSM)

- Two doublets to give masses to up-type and down-type fermions (extra symmetry forbids to use same doublet)

- SUSY imposes relations between the parameters

$\Rightarrow$ Two parameters instead of one: $\tan \beta \equiv \frac{v_{u}}{v_{d}}, \quad M_{\mathrm{A}}$ (or $M_{\mathrm{H}^{ \pm}}$)

$\Rightarrow$ Upper bound on lightest Higgs mass, $M_{\mathrm{h}}$ :

Lowest order: $M_{\mathrm{h}} \leq M_{\mathrm{Z}}$

Including higher-order corrections: $M_{\mathrm{h}} \lesssim 135 \mathrm{GeV}$ 


\section{Higgs physics in Supersymmetry}

"Simplest" extension of the minimal Higgs sector:

Minimal Supersymmetric Standard Model (MSSM)

- Two doublets to give masses to up-type and down-type fermions (extra symmetry forbids to use same doublet)

- SUSY imposes relations between the parameters

$\Rightarrow$ Two parameters instead of one: $\tan \beta \equiv \frac{v_{u}}{v_{d}}, \quad M_{\mathrm{A}}$ (or $M_{\mathrm{H}^{ \pm}}$)

$\Rightarrow$ Upper bound on lightest Higgs mass, $M_{\mathrm{h}}$ :

Lowest order: $M_{\mathrm{h}} \leq M_{\mathrm{Z}}$

Including higher-order corrections: $M_{\mathrm{h}} \lesssim 135 \mathrm{GeV}$

Detection of a SM-like Higgs with $M_{\mathrm{H}} \gtrsim 135 \mathrm{GeV}$ would have unambiguously ruled out the MSSM, signal at $\sim 126 \mathrm{GeV}$ is well compatible with MSSM prediction 
Future analyses: effective Lagrangian approach, obtained from integrating out heavy particles

Assumption: new physics appears only at a scale

$\Lambda \gg M_{\mathrm{h}} \sim 126 \mathrm{GeV}$

Systematic approach: expansion in inverse powers of $\Lambda$; parametrises deviations of coupling strenghts and tensor structure

$$
\Delta \mathcal{L}=\sum_{i} \frac{a_{i}}{\Lambda^{2}} \mathcal{O}_{i}^{d=6}+\sum_{j} \frac{a_{j}}{\Lambda^{4}} \mathcal{O}_{j}^{d=8}+\ldots
$$


Future analyses: effective Lagrangian approach, obtained from integrating out heavy particles

Assumption: new physics appears only at a scale

$\Lambda \gg M_{\mathrm{h}} \sim 126 \mathrm{GeV}$

Systematic approach: expansion in inverse powers of $\Lambda$; parametrises deviations of coupling strenghts and tensor structure

$$
\Delta \mathcal{L}=\sum_{i} \frac{a_{i}}{\Lambda^{2}} \mathcal{O}_{i}^{d=6}+\sum_{j} \frac{a_{j}}{\Lambda^{4}} \mathcal{O}_{j}^{d=8}+\ldots
$$

How about light BSM particles?

Difficult to incorporate in a generic way, need full structure of particular models 
Future analyses: effective Lagrangian approach, obtained from integrating out heavy particles

Assumption: new physics appears only at a scale

$\Lambda \gg M_{\mathrm{h}} \sim 126 \mathrm{GeV}$

Systematic approach: expansion in inverse powers of $\Lambda$; parametrises deviations of coupling strenghts and tensor structure

$$
\Delta \mathcal{L}=\sum_{i} \frac{a_{i}}{\Lambda^{2}} \mathcal{O}_{i}^{d=6}+\sum_{j} \frac{a_{j}}{\Lambda^{4}} \mathcal{O}_{j}^{d=8}+\ldots
$$

How about light BSM particles?

Difficult to incorporate in a generic way, need full structure of particular models

$\Rightarrow$ Analyses in terms of SM + effective Lagrangian and in specific BSM models: MSSM, ... are complementary 


\section{What does the signal at $\sim 126 \mathrm{GeV}$ tell us?}

Possible interpretations of the signal at $\sim 126 \mathrm{GeV}$ : 


\section{What does the signal at $\sim 126 \mathrm{GeV}$ tell us?}

Possible interpretations of the signal at $\sim 126 \mathrm{GeV}$ :

Option 0: A state that is not directly related to EWSB

Dilaton, ... 


\section{What does the signal at $\sim 126 \mathrm{GeV}$ tell us?}

Possible interpretations of the signal at $\sim 126 \mathrm{GeV}$ :

Option 0: A state that is not directly related to EWSB

Dilaton, ...

$\Rightarrow$ In principle a possible option, but looks increasingly unlikely

One would also expect to see other signatures of the EWSB dynamics in such a case soon ... 


\section{Option 1: "The" Standard Model Higgs}




\section{Option 1: "The" Standard Model Higgs}

What does this actually mean?

The SM is necessarily incomplete (does not include gravity, ...) 


\section{Option 1: "The” Standard Model Higgs}

What does this actually mean?

The SM is necessarily incomplete (does not include gravity, ...)

$\Rightarrow$ Interpretation in terms of "the" SM Higgs would imply that the low-energy limit of a more complete theory is just the $\mathrm{SM}+$ nothing else 


\section{Option 1: "The" Standard Model Higgs}

What does this actually mean?

The SM is necessarily incomplete (does not include gravity, ...)

$\Rightarrow$ Interpretation in terms of "the" SM Higgs would imply that the low-energy limit of a more complete theory is just the $\mathrm{SM}+$ nothing else

$\Rightarrow$ A logical possibility, but this would mean that the gauge hierarchy, dark matter, matter-anti-matter asymmetry in the universe, ..., would all have origins that are not directly related to low-scale physics 
Option 2: Higgs boson of an extended Higgs sector in the decoupling limit

Signal at $\sim 126 \mathrm{GeV}$ would correspond to the lightest Higgs state of an extended Higgs sector, all other Higgses heavy

Deviations from SM predictions for Higgs couplings are inversely proportional to the scale of the heavy Higgses

Example:

SUSY Higgs sector in the decoupling limit, $M_{\mathrm{A}} \gg M_{\mathrm{Z}}$ 


\section{MSSM fit (pre HCP): comparison of SM with}

\section{MSSM interpretation in terms of light Higgs $h$}

[P. Bechtle, S. Heinemeyer, O. Stål, T. Stefaniak, G. W., L. Zeune '12] -LHC / TeV. data, $\square$ full fit, $\square$ without TeV., $\diamond$ without low. en. obs.

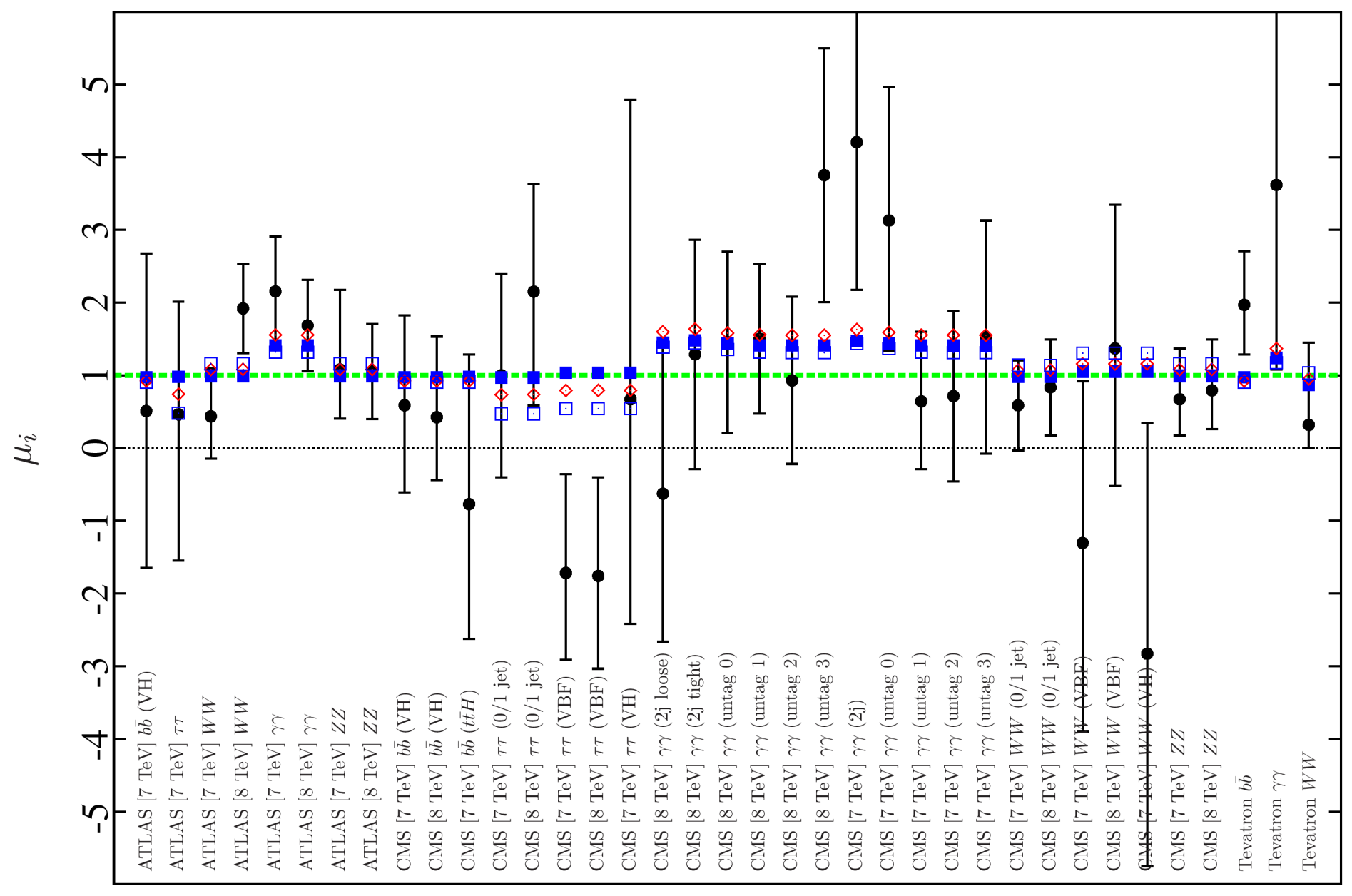

$\Rightarrow \chi^{2}$ reduced compared to SM case, better fit probability 


\section{Option 3: A mixed state or a composite Higgs}

- Mixed state Higgs-radion, ... 


\section{Option 3: A mixed state or a composite Higgs}

- Mixed state Higgs-radion, ...

- Composite "pseudo-Goldstone boson", like the pion in QCD $\Rightarrow$ Would imply new kind of strong interaction Relation to weakly-coupled 5-dimensional model (AdS/CFT correspondence) 


\section{Option 3: A mixed state or a composite Higgs}

- Mixed state Higgs-radion, ...

- Composite "pseudo-Goldstone boson", like the pion in QCD $\Rightarrow$ Would imply new kind of strong interaction Relation to weakly-coupled 5-dimensional model (AdS/CFT correspondence)

Discrimination from fundamental scalar

- Precision measurements of couplings $(\Rightarrow$ high sensitivity to compositeness scale), $\mathcal{C P}$ properties, ... Does the new state have the right properties to unitarize $W_{L} W_{L}$ scattering?

- Search for resonances (light Higgs $\Leftrightarrow$ light resonances?) 


\section{Option 4: The second-lightest Higgs of an extended Higgs sector}

Extended Higgs sector where the second-lightest Higgs at $\sim 126 \mathrm{GeV}$ has SM-like couplings to gauge bosons 


\section{Option 4: The second-lightest Higgs of an extended Higgs sector}

Extended Higgs sector where the second-lightest Higgs at $\sim 126 \mathrm{GeV}$ has SM-like couplings to gauge bosons

$\Rightarrow$ Lightest neutral Higgs with heavily suppressed couplings to gauge bosons, may have mass below the LEP limit of $M_{\mathrm{HSM}}>114.4 \mathrm{GeV}$ (in agreement with LEP bounds) 


\section{Option 4: The second-lightest Higgs of an extended Higgs sector}

Extended Higgs sector where the second-lightest Higgs at $\sim 126 \mathrm{GeV}$ has SM-like couplings to gauge bosons

$\Rightarrow$ Lightest neutral Higgs with heavily suppressed couplings to gauge bosons, may have mass below the LEP limit of $M_{\mathrm{H}_{\mathrm{SM}}}>114.4 \mathrm{GeV}$ (in agreement with LEP bounds)

Possible realisations: 2HDM, MSSM, NMSSM, ...

Example: "Low $M_{\mathrm{H}}$ benchmark scenario" of the MSSM 


\section{Option 4: The second-lightest Higgs of an extended Higgs sector}

Extended Higgs sector where the second-lightest Higgs at $\sim 126 \mathrm{GeV}$ has SM-like couplings to gauge bosons

$\Rightarrow$ Lightest neutral Higgs with heavily suppressed couplings to gauge bosons, may have mass below the LEP limit of $M_{\mathrm{H}_{\mathrm{SM}}}>114.4 \mathrm{GeV}$ (in agreement with LEP bounds)

Possible realisations: 2HDM, MSSM, NMSSM, ...

Example: "Low $M_{\mathrm{H}}$ benchmark scenario" of the MSSM

$\Rightarrow$ Observation of a SM-like signal at $\sim 126 \mathrm{GeV}$ provides a strong motivation to look for non SM-like Higgses elsewhere 


\section{Option 4: The second-lightest Higgs of an extended Higgs sector}

Extended Higgs sector where the second-lightest Higgs at $\sim 126 \mathrm{GeV}$ has SM-like couplings to gauge bosons

$\Rightarrow$ Lightest neutral Higgs with heavily suppressed couplings to gauge bosons, may have mass below the LEP limit of $M_{\mathrm{HSM}}>114.4 \mathrm{GeV}$ (in agreement with LEP bounds)

Possible realisations: 2HDM, MSSM, NMSSM, ...

Example: "Low $M_{\mathrm{H}}$ benchmark scenario" of the MSSM

$\Rightarrow$ Observation of a SM-like signal at $\sim 126 \mathrm{GeV}$ provides a strong motivation to look for non SM-like Higgses elsewhere

$\Rightarrow$ The best way of experimentally proving that the observed state is not the SM Higgs would be to find in addition (at least one) non-SM like Higgs! 
Low $M_{\mathrm{H}}$ benchmark scenario of the MSSM: signal

\section{interpreted as heavy CP-even Higgs $H$}

Approximate treatment of latest CMS limit included

[M. Carena, S. Heinemeyer, O. Stål, C. Wagner, G. W. '13]

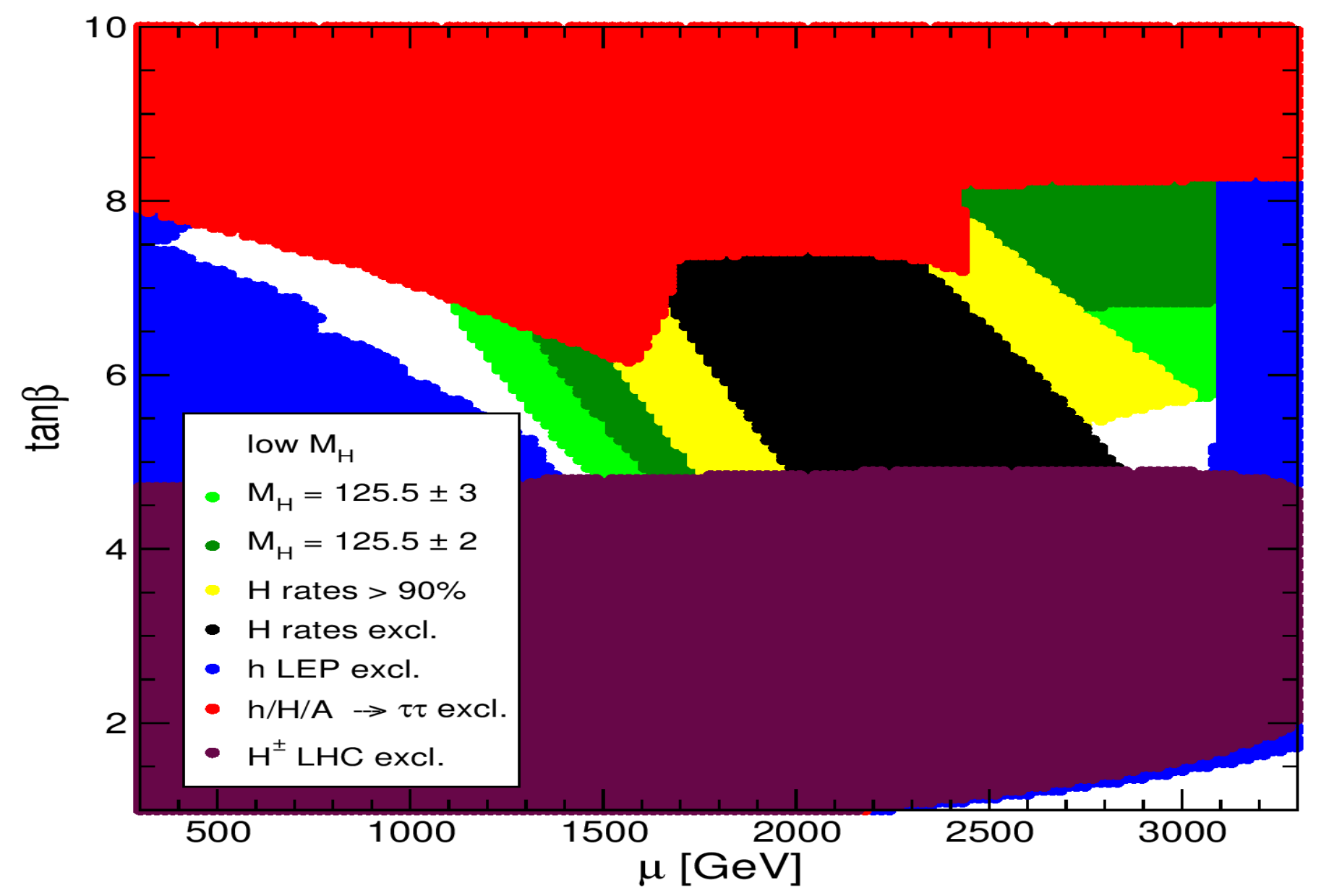

$\Rightarrow$ Rich phenomenology: all five MSSM Higgs bosons are light 
MSSM interpretation in terms of heavy Higgs $H$ :

\section{preferred values for $M_{\mathrm{H}^{ \pm}}$and $\mathrm{BR}\left(t \rightarrow H^{+} b\right)$}

[P. Bechtle, S. Heinemeyer, O. Stål, T. Stefaniak, G. W., L. Zeune '12]

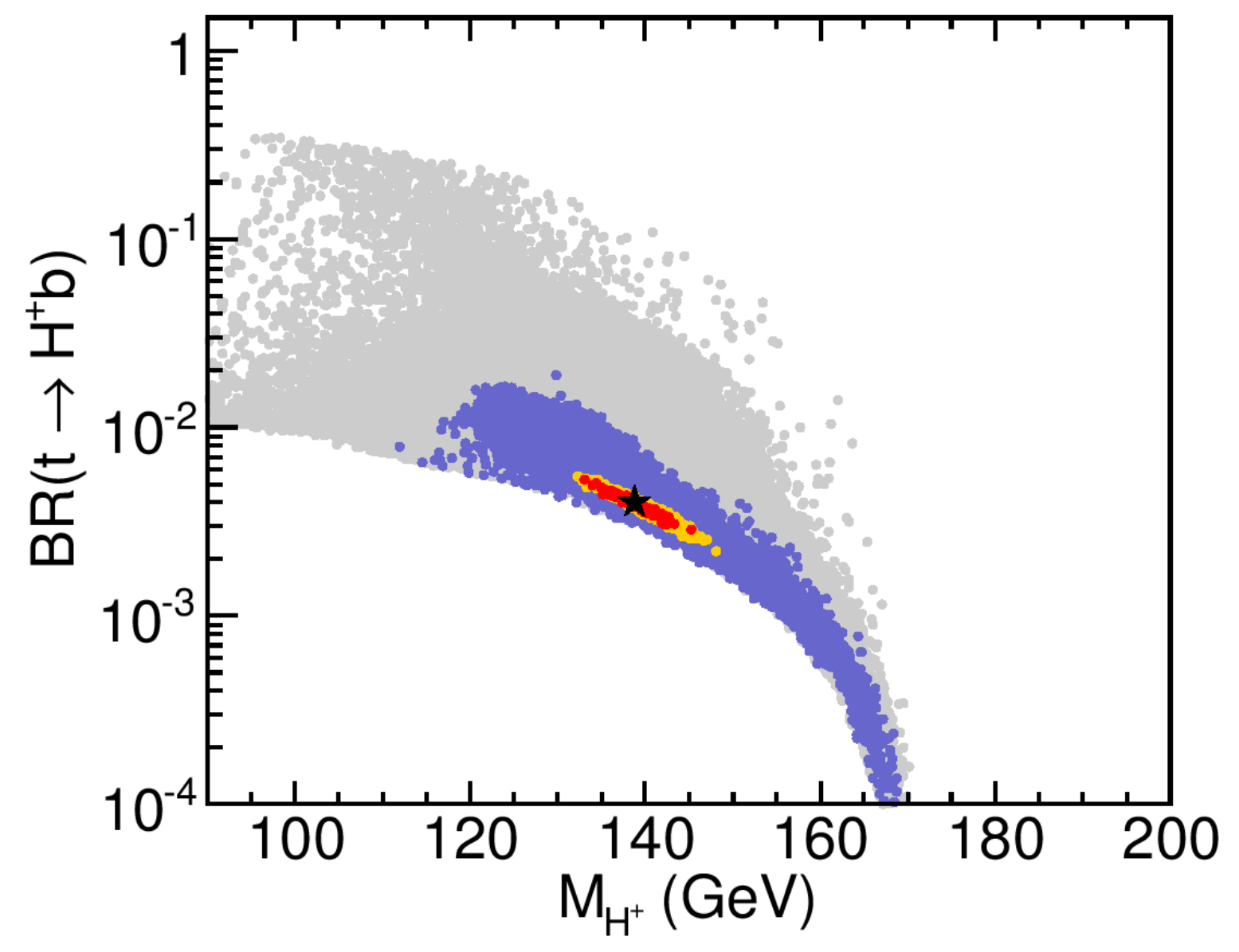

$\Rightarrow$ MSSM interpretation in terms of heavy Higgs $H$ can be probed by charged Higgs searches 


\section{Would such a light Higgs be detectable at the $H L-L H C$ and / or the ILC?}

- HL-LHC: 


\section{Would such a light Higgs be detectable at the $H L-L H C$ and / or the ILC?}

- HL-LHC:

- Not in decays of the state at $\sim 126 \mathrm{GeV}$ if mass of lightest Higgs $\gtrsim 63 \mathrm{GeV}$ 


\section{Would such a light Higgs be detectable at the $H L-L H C$ and / or the ILC?}

- HL-LHC:

- Not in decays of the state at $\sim 126 \mathrm{GeV}$ if mass of lightest Higgs $\gtrsim 63 \mathrm{GeV}$

- So far there are no LHC searches for light Higgses in this mass range 


\section{Would such a light Higgs be detectable at the $H L-L H C$ and / or the ILC?}

- HL-LHC:

- Not in decays of the state at $\sim 126 \mathrm{GeV}$ if mass of lightest Higgs $\gtrsim 63 \mathrm{GeV}$

- So far there are no LHC searches for light Higgses in this mass range

- In case of SUSY, such a light Higgs could be produced in a SUSY cascade, e.g. $\tilde{\chi}_{2}^{0} \rightarrow \tilde{\chi}_{1}^{0} h$; could be similar for other types of BSM physics 


\section{Would such a light Higgs be detectable at the $H L-L H C$ and / or the ILC?}

- HL-LHC:

- Not in decays of the state at $\sim 126 \mathrm{GeV}$ if mass of lightest Higgs $\gtrsim 63 \mathrm{GeV}$

- So far there are no LHC searches for light Higgses in this mass range

- In case of SUSY, such a light Higgs could be produced in a SUSY cascade, e.g. $\tilde{\chi}_{2}^{0} \rightarrow \tilde{\chi}_{1}^{0} h$; could be similar for other types of BSM physics

- ILC:

- Pair production, e.g. SUSY case: $e^{+} e^{-} \rightarrow h A$ $(+t \bar{t} h$ production, ....) 


\section{Thus, where do we stand?}




\section{Thus, where do we stand?}

What we know so far about the new state at $\sim 126 \mathrm{GeV}$ still leaves open many possible interpretations 


\section{Thus, where do we stand?}

What we know so far about the new state at $\sim 126 \mathrm{GeV}$ still leaves open many possible interpretations

ILC ("Higgs factory") measurements will be crucial for distinguishing between options $0,1,2,3,4$ :

- High-precision measurements of couplings to gauge bosons and fermions and of total width without theoretical assumptions

- Decay-mode independent measurement using "recoil" against the $Z$

$\Rightarrow$ Absolute measurement of couplings and total Higgs width, model-independent

- Higgs self-coupling $\Leftrightarrow$ experimental access to Higgs potential 


\section{ILC: high-precision measurements of \\ Higgs properties}

"Recoil" method: $e^{+} e^{-} \rightarrow Z H, Z \rightarrow e^{+} e^{-}, \mu^{+} \mu^{-}$[R. Poeschl et al. '12]

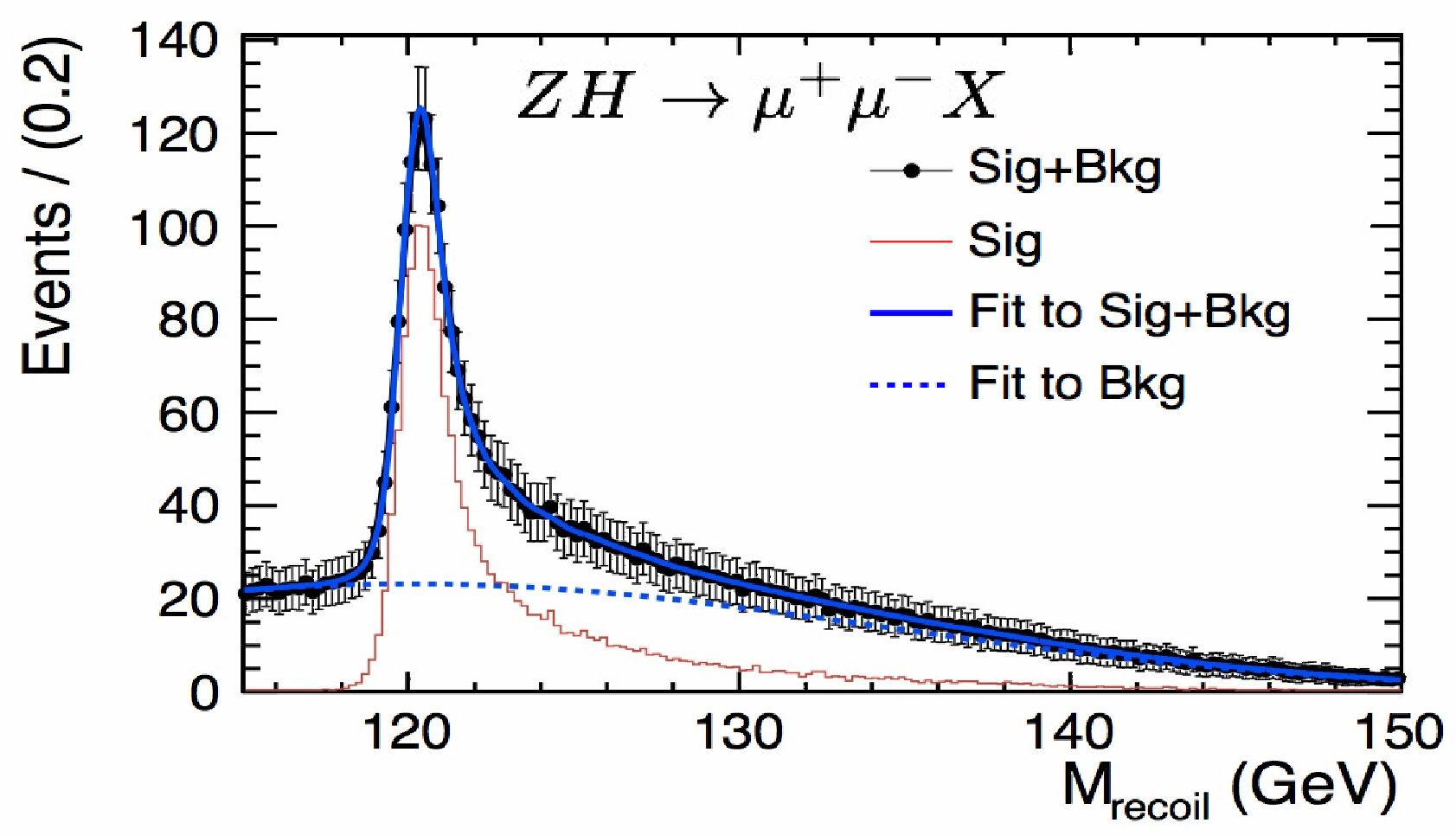

Measurement of mass, couplings, $\mathcal{C P}$ properties, self-coupling, ...+ high sensitivity to additional Higgses

$\Rightarrow$ Identification of the underlying nature of electroweak symmetry breaking 


\section{The quest for identifying the underlying physics}

Discrimination between different kinds of underlying physics via precision measurements of Higgs couplings
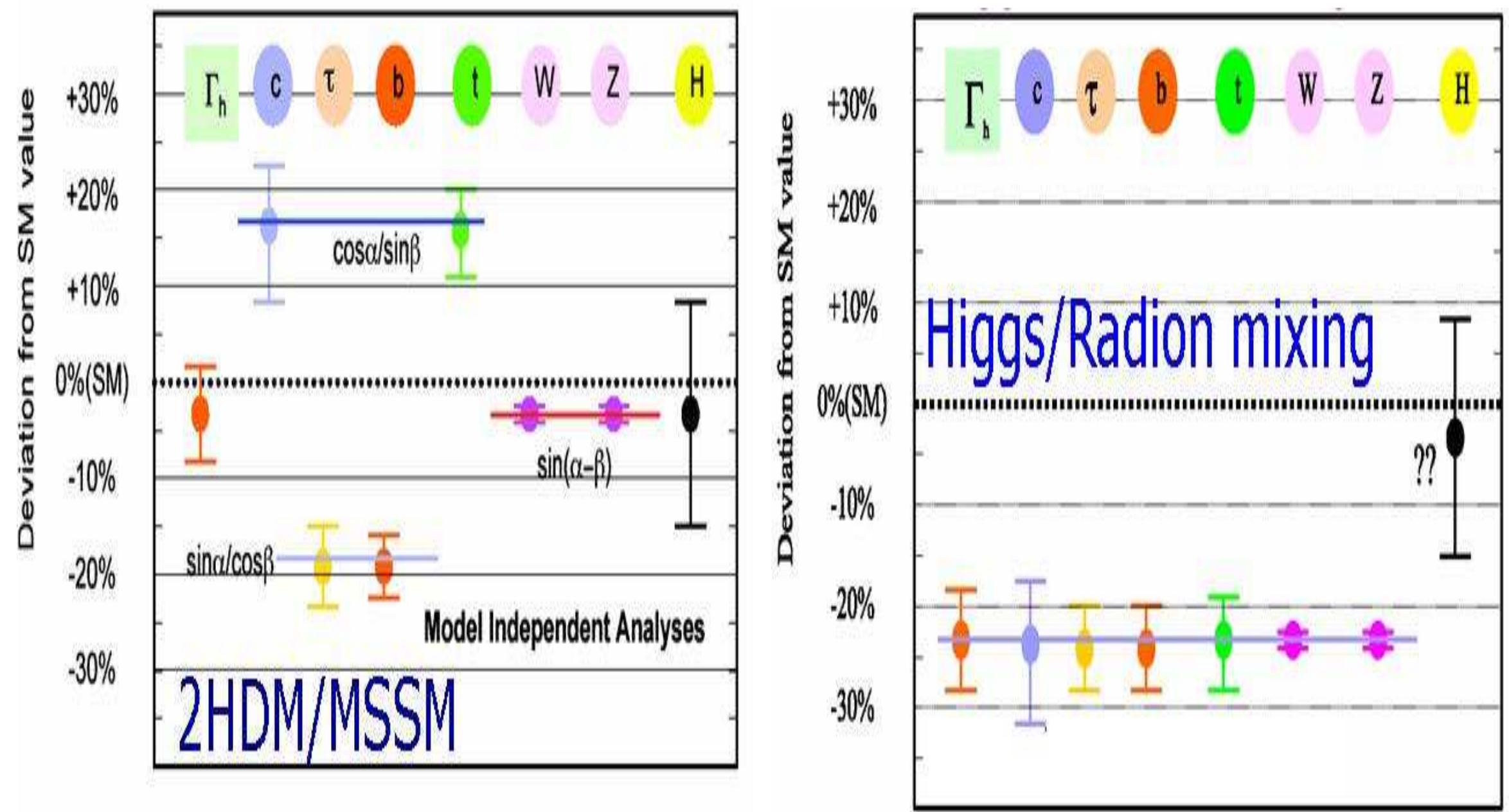


\section{Where is the new physics that stabilises the gauge hierarchy?}

Large number of searches, many limits, ..

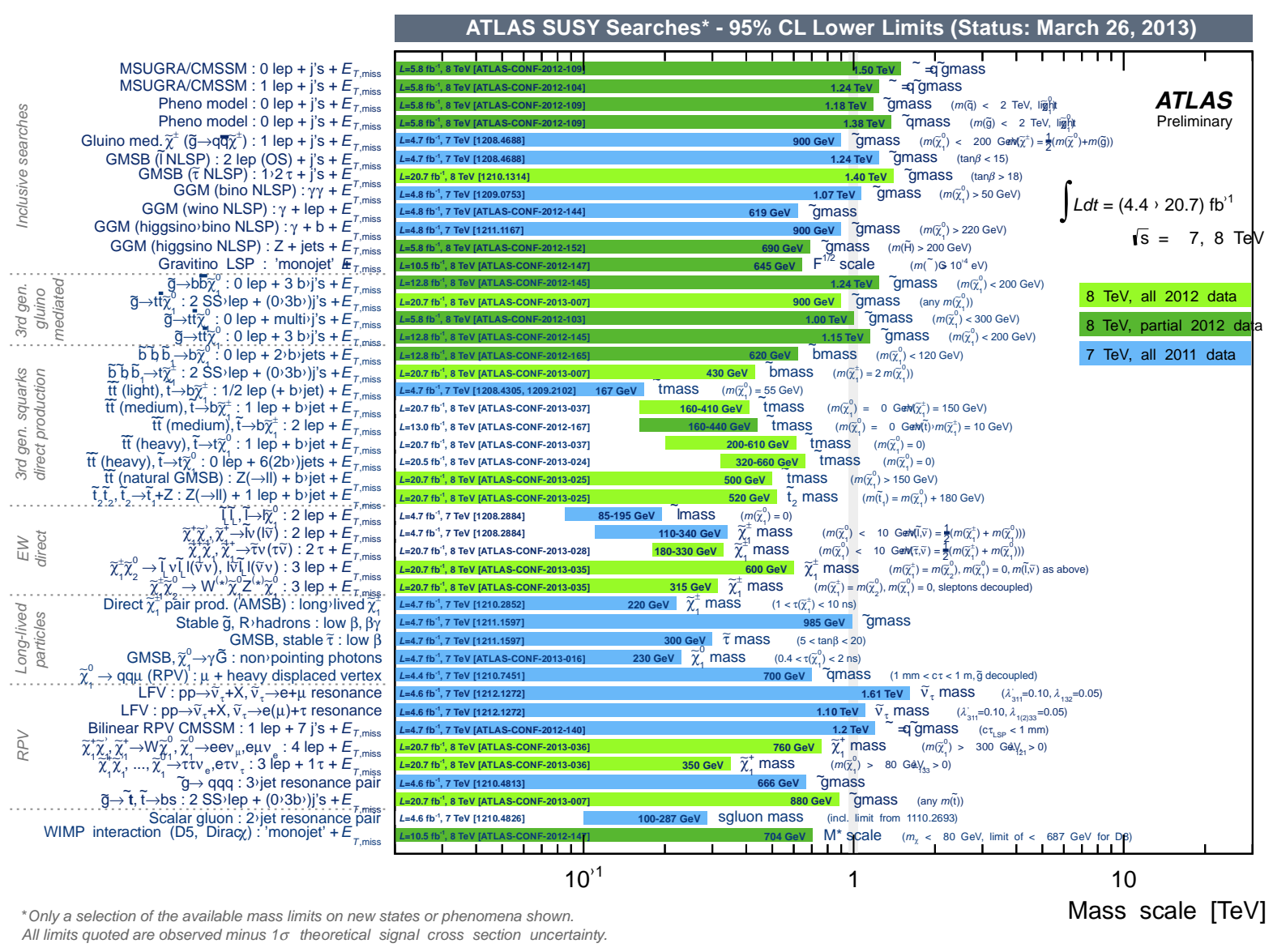

Interpretation in specific scenarios, e.g. CMSSM, and in "simplified models" 


\section{Nothing else but the Standard Model?}

Can the SM be valid all the way up to the Planck scale? 


\section{Nothing else but the Standard Model?}

Can the SM be valid all the way up to the Planck scale?

Yes, in principle, but ... 


\section{Nothing else but the Standard Model?}

Can the SM be valid all the way up to the Planck scale?

Yes, in principle, but ...

Do we live in a metastable vacuum?

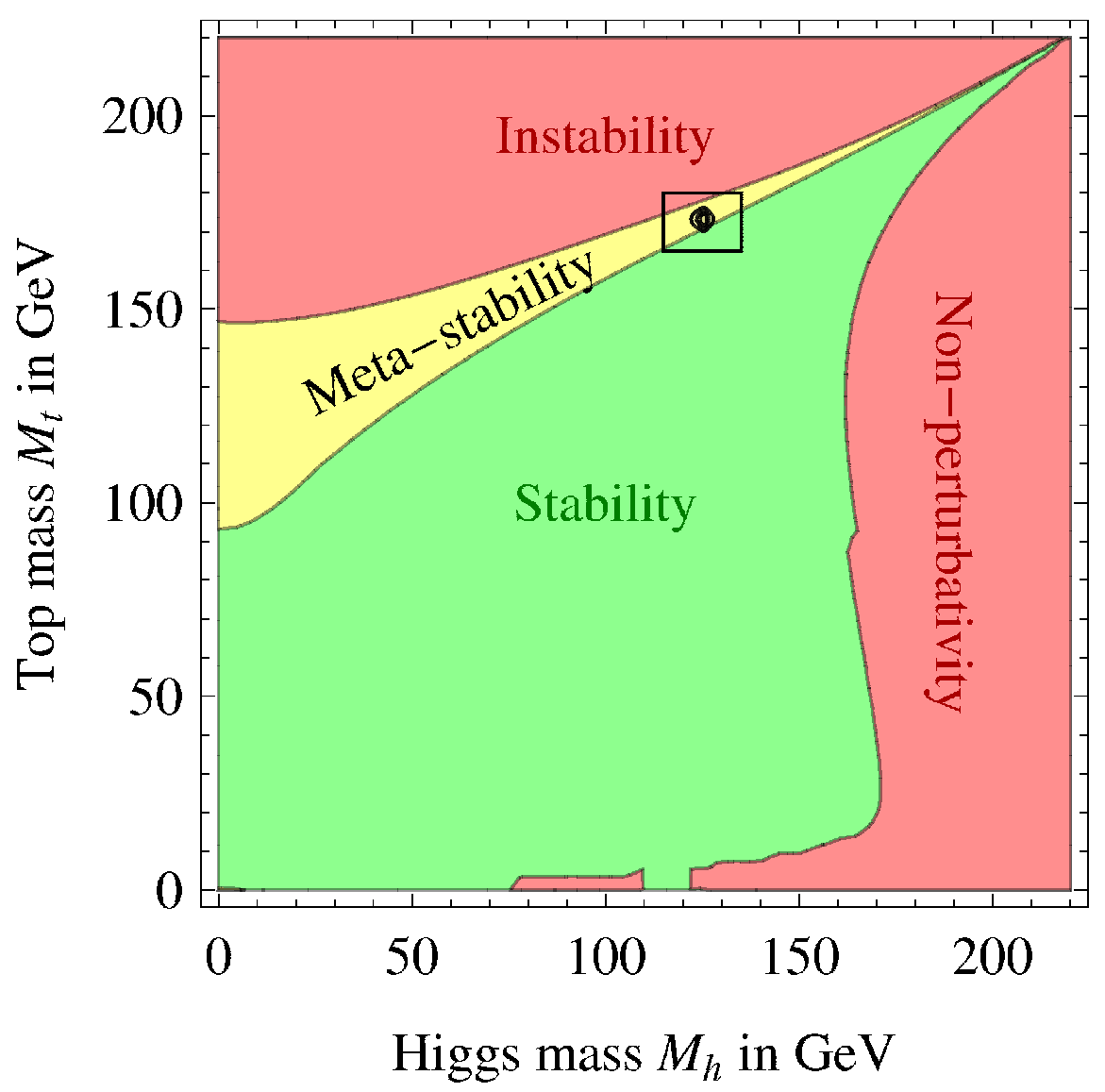

[G. Degrassi et al. '12] 
The hierarchy problem: SM Higgs mass is affected by large corrections $\left(\sim \Lambda^{2}\right)$ from physics at high scales

Now that a Higgs-like state with a mass of $\sim 126 \mathrm{GeV}$ has been discovered, the question what protects its mass from physics at high scale becomes even more pressing

"Hierarchy problem": $M_{\text {Planck }} / M_{\text {weak }} \approx 10^{17}$

How can two so different scales coexist in nature?

Via quantum effects: physics at $M_{\text {weak }}$ is affected by physics at $M_{\text {Planck }} \Rightarrow$ Instability of $M_{\text {weak }}$, would imply that all physics is driven up to the Planck scale

$\Rightarrow$ Expect new physics to stabilise the hierarchy

E.g. SUSY: Large corrections cancel out because of symmetry fermions $\Leftrightarrow$ bosons 


\section{What has actually been excluded?}

\section{Let's consider the old SPS 1a benchmark point}

A look back to the pre-LHC days:

Global fits in constrained SUSY models (CMSSM, ...):

Best fit point was close to SPS 1a $(\mathrm{LM} 1, \ldots)$ benchmark point:

Low scale SUSY point

$\Rightarrow$ "plain vanilla" SUSY

$\Rightarrow$ "best case scenario" for LHC and LC 


\section{What has actually been excluded?}

\section{Let's consider the old SPS 1a benchmark point}

A look back to the pre-LHC days:

Global fits in constrained SUSY models (CMSSM, ...):

Best fit point was close to SPS 1a $(\mathrm{LM} 1, \ldots)$ benchmark point:

Low scale SUSY point

$\Rightarrow$ "plain vanilla" SUSY

$\Rightarrow$ "best case scenario" for LHC and LC

Preference for light SUSY scale was mainly driven by $(g-2)_{\mu}$

$\Rightarrow$ light $\tilde{e}, \tilde{\mu}, \tilde{\chi}, \ldots$ : light electroweak SUSY particles 


\section{Particle spectrum of the SPS 1a benchmark point}

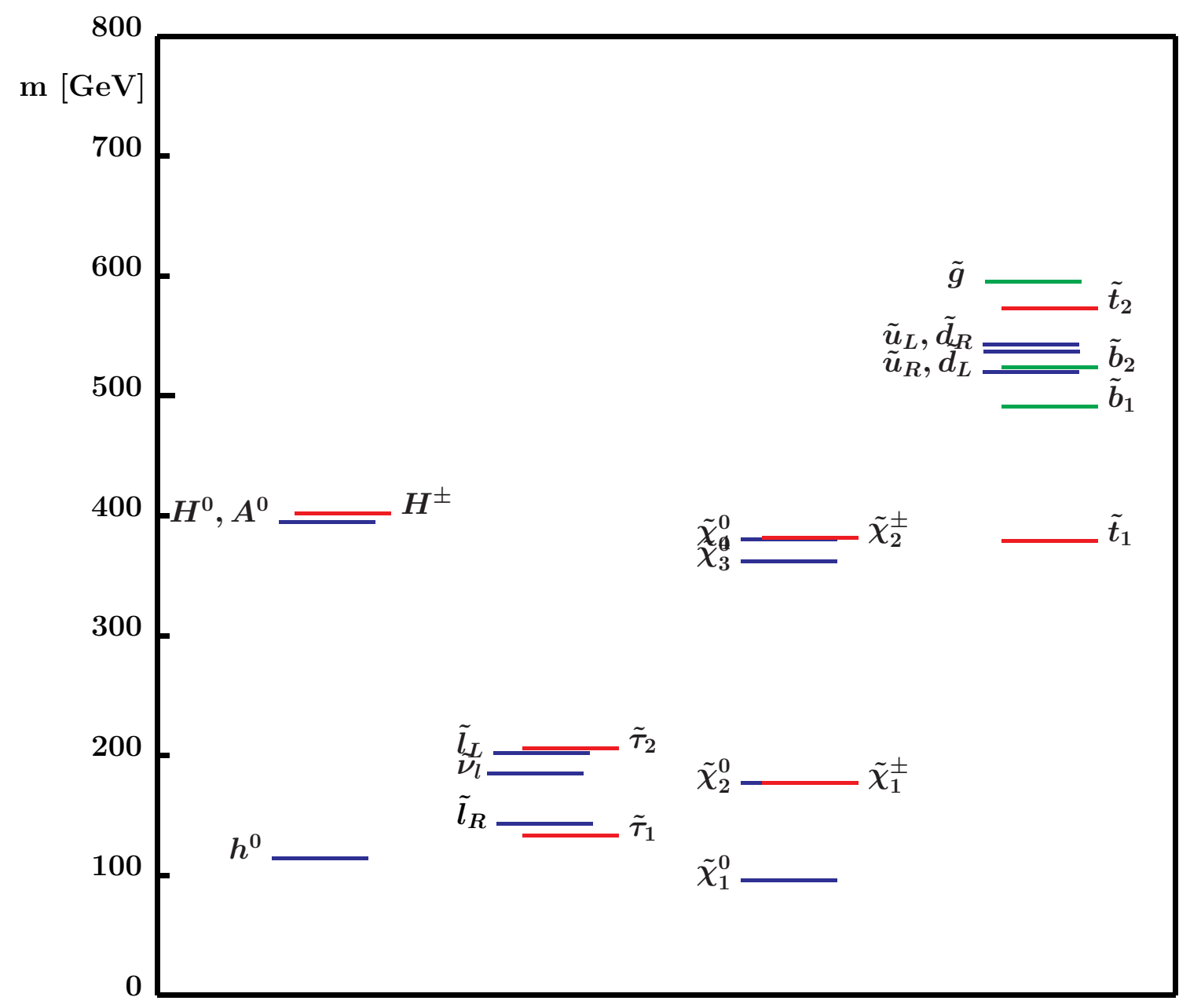

$\Rightarrow$ all SUSY masses below $600 \mathrm{GeV}$

$\Rightarrow$ "plain vanilla" SUSY at its best 


\section{My favourite question to ATLAS and CMS SUSY hunters}

Sensitivity for exclusion limits obtained at ATLAS and CMS so far relies mainly on the (strong interaction) production of the gluino and the squarks of the first two generations 


\section{My favourite question to ATLAS and CMS SUSY hunters}

Sensitivity for exclusion limits obtained at ATLAS and CMS so far relies mainly on the (strong interaction) production of the gluino and the squarks of the first two generations

Assume nature had chosen the SPS 1a spectrum, except that gluino and squarks of the first two generations were heavy

$\Rightarrow$ The masses of all other SUSY particles are at the "plain vanilla" SPS 1a benchmark values 


\section{My favourite question to ATLAS and CMS SUSY hunters}

Sensitivity for exclusion limits obtained at ATLAS and CMS so far relies mainly on the (strong interaction) production of the gluino and the squarks of the first two generations

Assume nature had chosen the SPS 1a spectrum, except that gluino and squarks of the first two generations were heavy

$\Rightarrow$ The masses of all other SUSY particles are at the "plain vanilla" SPS 1a benchmark values

\section{Question:}

Is it possible to exclude such a scenario with the present data

- from direct production of third generation squarks?

- from direct production of electroweak SUSY particles? 


\section{Sensitivity to effects of new physics from}

\section{high-precision measurements at the ILC}

- Measurements in the Higgs sector

- Top physics

- Electroweak precision observables $\left(\sin ^{2} \theta_{\mathrm{eff}}, M_{\mathrm{W}}, \ldots\right)$

Example: $M_{\mathrm{W}}-m_{\mathrm{t}}$ correlation

ILC:

- High-precision measurement of $m_{\mathrm{t}}$, relation between measured quantity and theoretically well-defined parameter is known with sufficient accuracy

- Precise measurement of $M_{\mathrm{W}}$ (continuum and $W W$ threshold) 


\section{Example: prediction for $M_{\mathrm{W}}$ (parameter scan),}

SM vs. MSSM (signal interpreted as light CP-even Higgs, $h$ )

Prediction for $M_{\mathrm{W}}$ in the SM and the MSSM:

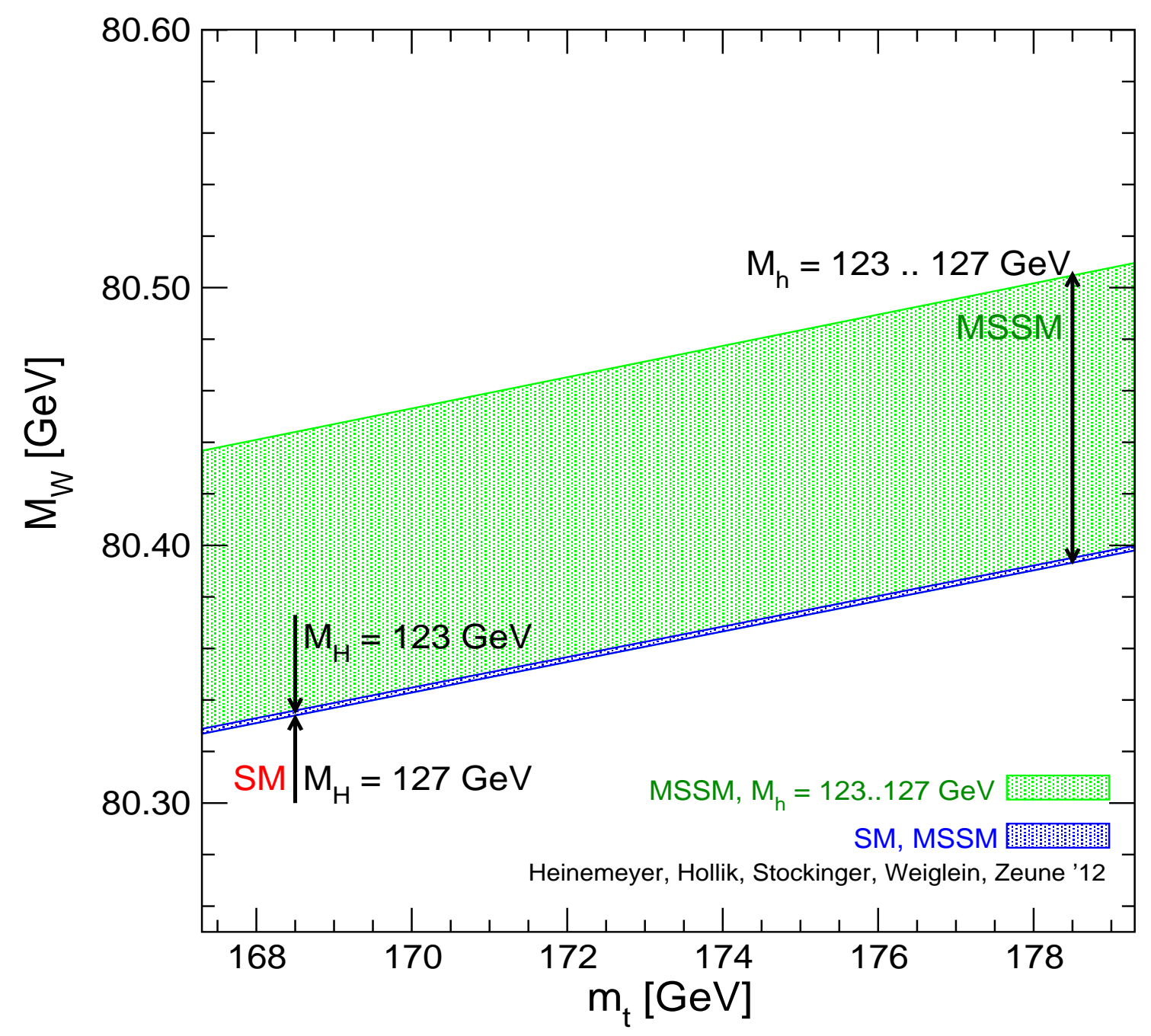

[S. Heinemeyer, W. Hollik,

D. Stöckinger, G. W.,

L. Zeune '12]

MSSM: SUSY parameters varied

SM: $M_{\mathrm{H}}$ varied

Tevatron result for $m_{\mathrm{t}}$ interpreted (perturb.) as pole mass 
Example: prediction for $M_{\mathrm{W}}$ (parameter scan),

SM vs. MSSM (signal interpreted as light CP-even Higgs, $h$ )

Prediction for $M_{\mathrm{W}}$ in the SM and the MSSM:

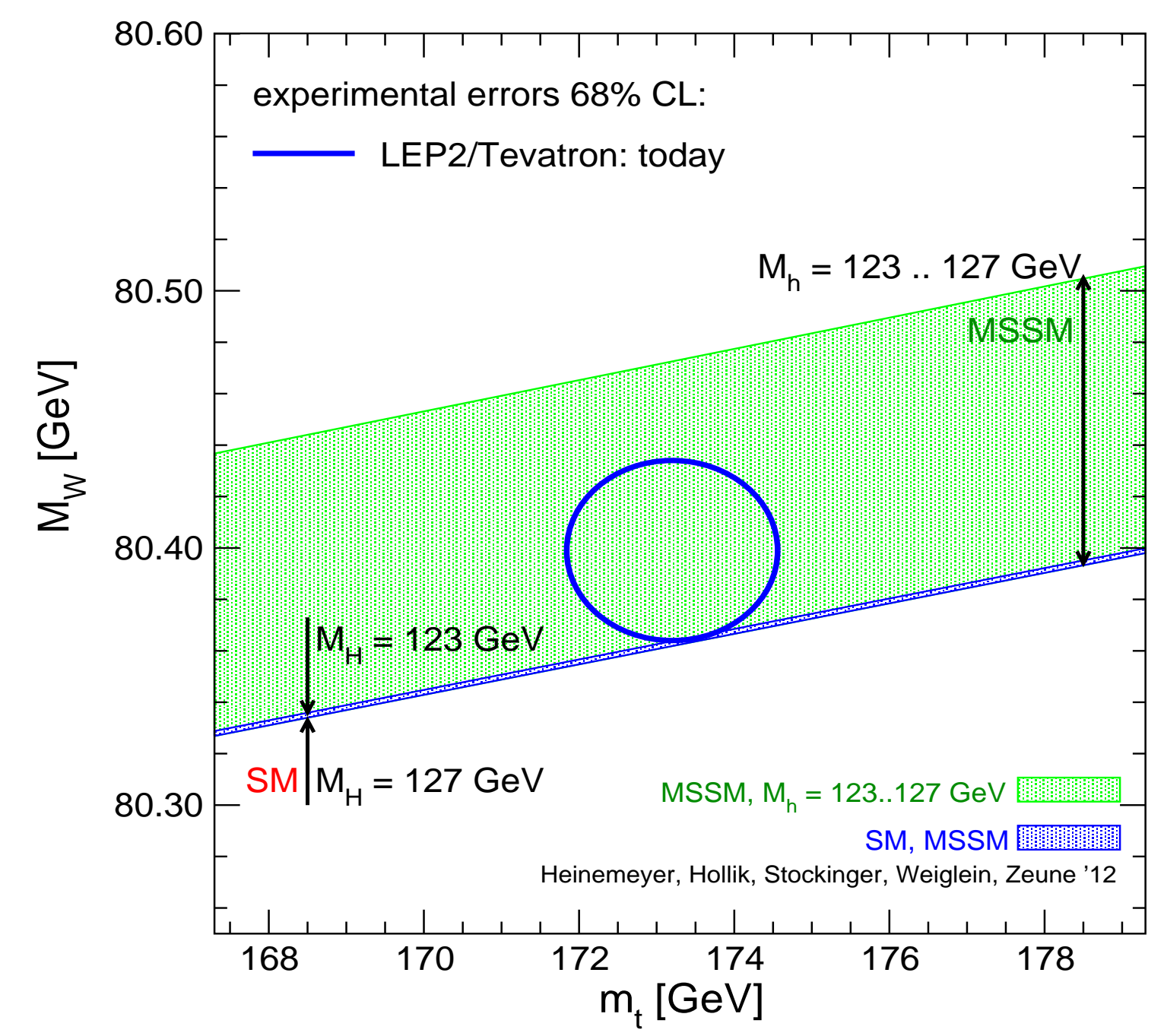

[S. Heinemeyer, W. Hollik,

D. Stöckinger, G. W.,

L. Zeune '12]

MSSM: SUSY parameters varied

SM: $M_{\mathrm{H}}$ varied

Tevatron result for $m_{\mathrm{t}}$ interpreted (perturb.) as pole mass

$\Rightarrow$ Slight preference for MSSM over SM 
Example: prediction for $M_{\mathrm{W}}$ (parameter scan),

SM vs. MSSM (signal interpreted as light CP-even Higgs, $h$ )

Prediction for $M_{\mathrm{W}}$ in the SM and the MSSM:

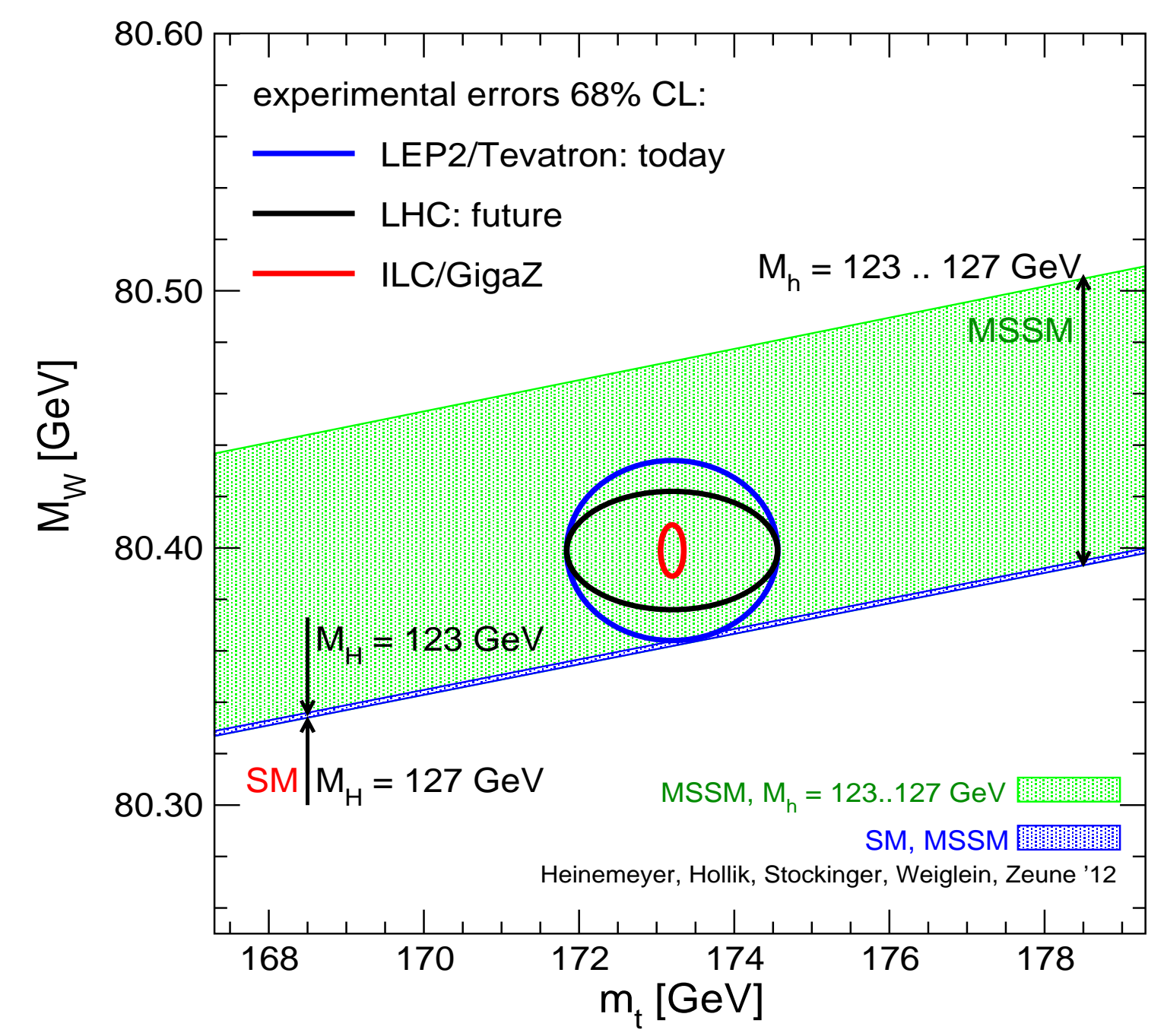

[S. Heinemeyer, W. Hollik,

D. Stöckinger, G. W.,

L. Zeune '12]

MSSM: SUSY parameters varied

SM: $M_{\mathrm{H}}$ varied

Tevatron result for $m_{\mathrm{t}}$ interpreted (perturb.) as pole mass

$\Rightarrow$ Slight preference for MSSM over SM 


\section{Example: prediction for $M_{\mathrm{W}}$ (parameter scan),}

\section{SM vs. MSSM (signal interpr. as heavy CP-even Higgs, $H$ )}

Prediction for $M_{\mathrm{W}}$ in the SM and the MSSM:

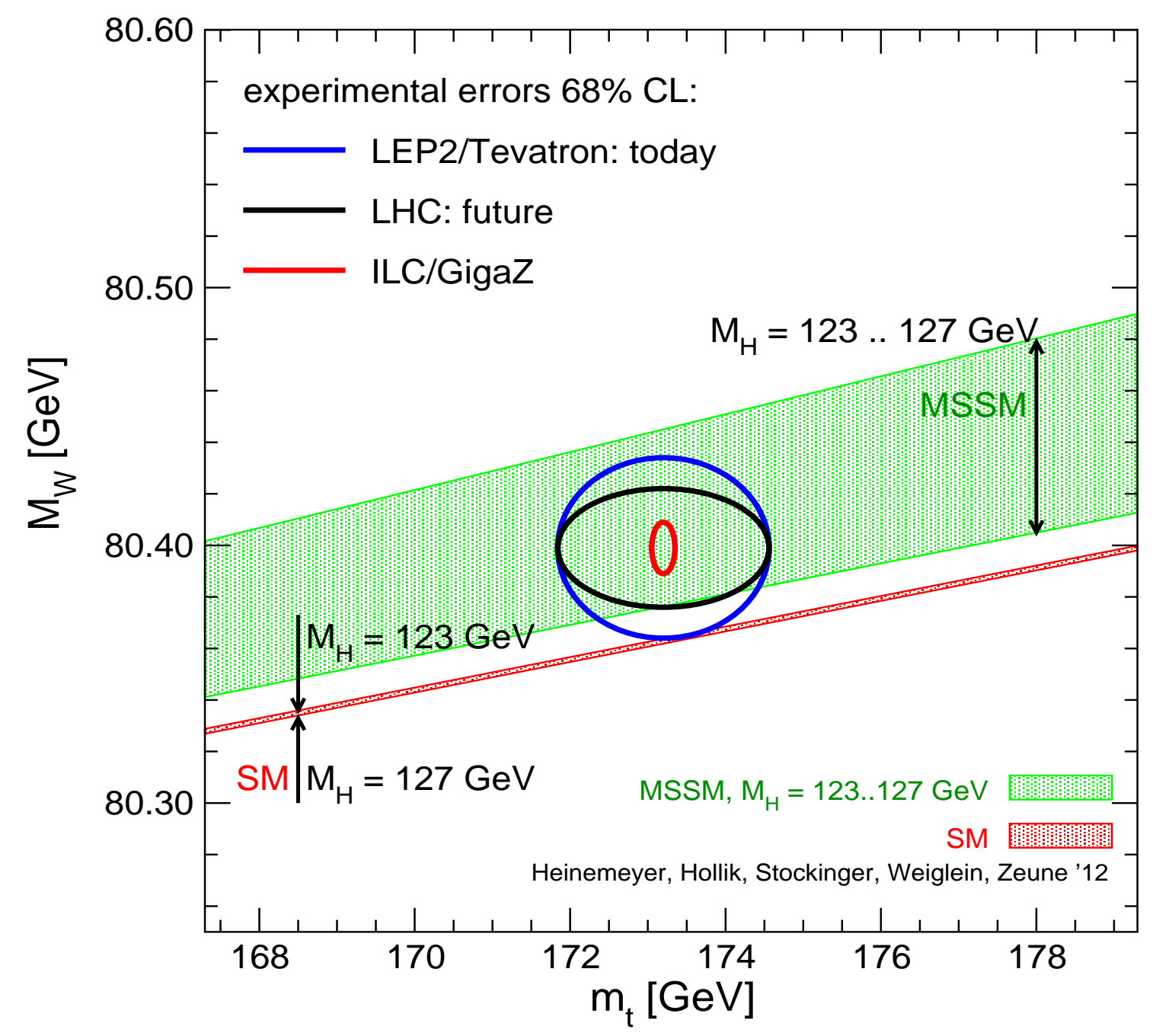

[S. Heinemeyer, W. Hollik,

D. Stöckinger, G. W.,

L. Zeune '12]

MSSM: SUSY parameters varied

SM: $M_{\mathrm{H}}$ varied

Tevatron result for $m_{\mathrm{t}}$ interpreted (perturb.) as pole mass

$\Rightarrow$ High sensitivity to possible effects of new physics 


\section{Conclusions}

- The signal at $\sim 126 \mathrm{GeV}$ is compatible with a SM-like Higgs, but a variety of interpretations are possible, correspond to very different underlying physics 


\section{Conclusions}

- The signal at $\sim 126 \mathrm{GeV}$ is compatible with a SM-like Higgs, but a variety of interpretations are possible, correspond to very different underlying physics

- ILC: ideal tool for identifying the nature of EWSB 


\section{Conclusions}

- The signal at $\sim 126 \mathrm{GeV}$ is compatible with a SM-like Higgs, but a variety of interpretations are possible, correspond to very different underlying physics

- ILC: ideal tool for identifying the nature of EWSB

- HL-LHC: Analyses of prospects (Higgs couplings, search for BSM Higgses, $W W$ scattering, ... ) should be as realistic as possible w.r.t. the physics scenario and the experimental conditions (pile-up, ...) 


\section{Conclusions}

- The signal at $\sim 126 \mathrm{GeV}$ is compatible with a SM-like Higgs, but a variety of interpretations are possible, correspond to very different underlying physics

- ILC: ideal tool for identifying the nature of EWSB

- HL-LHC: Analyses of prospects (Higgs couplings, search for BSM Higgses, $W W$ scattering, ... ) should be as realistic as possible w.r.t. the physics scenario and the experimental conditions (pile-up, ...)

- Good prospects for discovering BSM particles at LHC and ILC 


\section{Conclusions}

- The signal at $\sim 126 \mathrm{GeV}$ is compatible with a SM-like Higgs, but a variety of interpretations are possible, correspond to very different underlying physics

- ILC: ideal tool for identifying the nature of EWSB

- HL-LHC: Analyses of prospects (Higgs couplings, search for BSM Higgses, $W W$ scattering, ... ) should be as realistic as possible w.r.t. the physics scenario and the experimental conditions (pile-up, ...)

- Good prospects for discovering BSM particles at LHC and ILC

- Precision measurements provide high indirect sensitivity for effects of new physics 